\title{
Van der Waals junction field effect transistors with both n- and p-channel transition metal dichalcogenides
}

\author{
June Yeong Lim ${ }^{1}$, Minju Kim (D) ${ }^{1}$, Yeonsu Jeong ${ }^{1}$, Kyeong Rok Ko ${ }^{1}$, Sanghyuck Yu ${ }^{1}$, Hyung Gon Shin ${ }^{1}$, Jae Young Moon ${ }^{1}$, Young Jai Choi ${ }^{1}$,
} Yeonjin $\mathrm{Yi}^{1}$, Taekyeong $\mathrm{Kim}^{2}$ and Seongil $\mathrm{Im}^{1}$

Two-dimensional (2D) transition metal dichalcogenides (TMDs)-based van der Waals (vdW) PN junctions have been used for heterojunction diodes, which basically utilize out-of-plane current across the junction interface. In fact, the same vdW PN junction structure can be utilized for another important device application, junction field effect transistors (JFETs), where in-plane current is possible along with 2D-2D heterojunction interface. Moreover, the 2D TMD-based JFET can use both $p$ - and $n$-channel for low voltage operation, which might be its unique feature. Here we report vdW JFETs as an in-plane current device with heterojunction between semiconducting $\mathrm{p}$ - and $\mathrm{n}$-TMDs. Since this vdW JFET would have low-density traps at the vdW interface unlike 2D TMDbased metal insulator semiconductor field effect transistors (MISFETs), little hysteresis of 0.0-0.1 V and best subthreshold swing of $\sim 100 \mathrm{mV} / \mathrm{dec}$ were achieved. Easy saturation was observed either from $\mathrm{n}$-channel or $\mathrm{p}$-channel JFET as another advantage over 2D MISFETs, exhibiting early pinch-off at $\sim 1 \mathrm{~V}$. Operational gate voltage for threshold was near $0 \mathrm{~V}$ and our highest mobility reaches to $\sim 500 \mathrm{~cm}^{2} \mathrm{~V} \cdot \mathrm{s}$ for $\mathrm{n}$-channel JFET with $\mathrm{MoS}_{2}$ channel. For $1 \mathrm{~V}$ JFET operation, our best ON/OFF current ratio was observed to be $\sim 10^{4}$.

npj 2D Materials and Applications (2018)2:37 ; doi:10.1038/s41699-018-0082-2

\section{INTRODUCTION}

Two-dimensional (2D) transition metal dichalcogenides (TMDs) have been extensively studied in both aspects of materials and devices for the past decade, since those are regarded to have great potentials for future nanoelectronics. ${ }^{1-11}$ Main focus has been on 2D TMD semiconductor devices, which are probably the most important for existing or future technologies..$^{3,8-12}$ Many of metal insulator semiconductor field effect transistors (MISFETs) using 2D TMD channels have thus been reported, ${ }^{8,10,13-22}$ along with their use for complementary metal-oxide-semiconductor transistor inverters. ${ }^{23-28}$ Heterojunction 2D TMD $\mathrm{p}-\mathrm{n}(\mathrm{PN})$ diodes with van der Waals (vdW) interface have also received much attention from researchers. ${ }^{29-44}$ These vdW PN junction interfaces basically experience out-of-plane or vertical current across the junction during device operation. In fact, the same vdW PN junction structure can be utilized for another important device application, junction field effect transistors (JFETs), where in-plane current is possible along with 2D-2D heterojunction interface. However, the vdW JFET application seems not reported yet, although some possibilities have just been casted in black phosphorous/ZnO nanowire junction systems. ${ }^{45}$

In the present work, we have fabricated vdW JFETs as an inplane current device with heterojunction between semiconducting $\mathrm{p}-\mathrm{MoTe}_{2}$ (or $\mathrm{p}-\mathrm{WSe}_{2}$ ) and $\mathrm{n}-\mathrm{MoS}_{2}$ TMDs. Since this vdW JFET would have low-density traps at the heterojunction interface when $\mathrm{p}$-type material plays as a gate for $\mathrm{n}$-channel and vice versa, little hysteresis of $0.05-0.1 \mathrm{~V}$ and good subthreshold swing (SS) of $\sim 100 \mathrm{mV} / \mathrm{dec}$ were achieved. In addition, vdW JFET always exhibited easy saturation at a low drain voltage of $\sim 1 \mathrm{~V}$ and reproducibly showed low operational gate voltages for threshold near $0 \mathrm{~V}(+0.2 \mathrm{~V}$ for $\mathrm{p}-\mathrm{JFET}$ and $-0.2 \mathrm{~V}$ for $\mathrm{n}-\mathrm{JFET})$. Abovementioned properties have rarely been realized all together from general vdW 2D MISFETs. ${ }^{20,22}$ The highest mobility reaches to $\sim 500 \mathrm{~cm}^{2} / \mathrm{V}$.s for $\mathrm{n}$-channel JFET with $\mathrm{MoS}_{2}$ channel while $\mathrm{p}$ channel JFET with $\mathrm{MoTe}_{2}$ appears much lower by more than an order of magnitude $\left(\sim 13 \mathrm{~cm}^{2} / \mathrm{V} \cdot \mathrm{s}\right)$. These values are comparable or approaches to previous results from 2D FETs. ${ }^{12,25,46}$ For our lowvoltage JFET devices, ON/OFF current ratios were observed to be $\sim 10^{4}$. The operation of both channel JFETs with ultrathin vdW 2D TMDs is regarded unique and different from that of general threedimensional (3D) JFETs and MISFETs, and in principle two opposite ( $p$ and $n$ ) channels can be used as gate for each other. We again confirmed the principle through another $p-T M D / n-T M D$ JFET ( $p$ WSe 2 /n-MoS 2 junction).

\section{RESULTS AND DISCUSSION}

Figure 1a displays an optical microscopic (OM) image of our $\mathrm{p}$ $\mathrm{MoTe}_{2} / \mathrm{n}-\mathrm{MoS}_{2}$ channel JFET device fabricated on 285-nm-thick $\mathrm{SiO}_{2} / \mathrm{p}$-Si wafer. Pt and $\mathrm{Au}$ are used as source/drain ohmic electrodes, respectively, for $\mathrm{MoTe}_{2}$ and $\mathrm{MoS}_{2}$ channel. As shown in a $3 \mathrm{D}$ schematic device view in Fig. $1 \mathrm{c}$, hexagonal $2 \mathrm{H}-\mathrm{MoS}_{2}$ channel overlies on $2 \mathrm{H}-\mathrm{MoTe}_{2}$ when the two channels cross each other. The two semiconducting TMDs are simultaneously and clearly identified by micro Raman spectroscopy as shown in Fig. 1b, for which a central spot of the overlapped region is probed (see the red spot in Fig. 1a). Since the p- and n-channel materials are crossing, four different $\mathrm{PN}$ diodes are possibly formed using $\mathrm{Pt}$ and $\mathrm{Au}$ electrode and such diode behavior was confirmed (see

\footnotetext{
${ }^{1}$ Van der Waals Materials Research Center, Yonsei University, 50 Yonsei-ro, Seodaemun-gu, Seoul 03722, Korea and ${ }^{2}$ Department of Physics, Hankuk University of Foreign Studies, 81 Oedae-ro, Chein-gu, Yongin-si 17035, South Korea

Correspondence: Seongil Im (semicon@yonsei.ac.kr)
}

Received: 22 June 2018 Revised: 10 October 2018 Accepted: 15 October 2018

Published online: 05 November 2018 
(a)

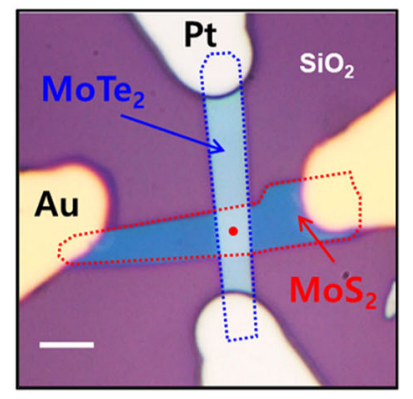

(c)

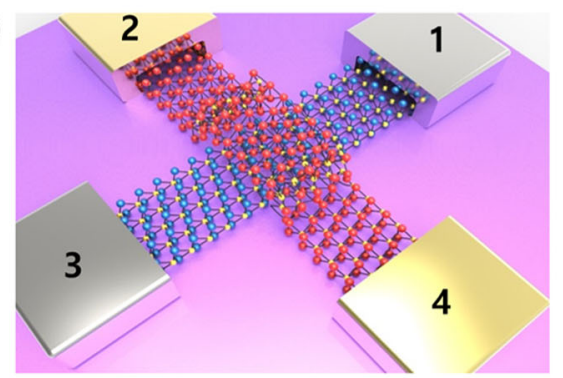

(d)

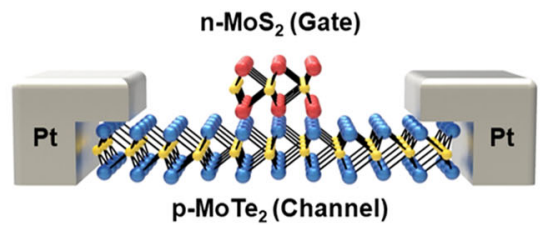

(b)
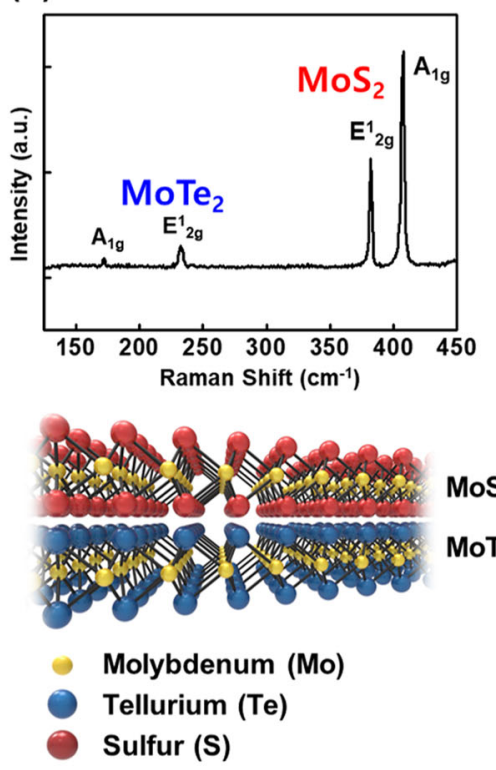

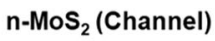

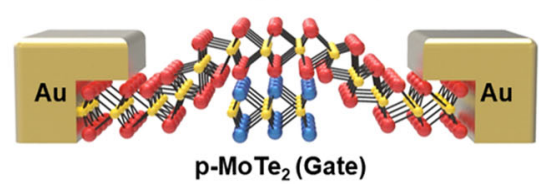

Fig. 1 Materials characteristics and device schematic views. a Optical microscopic (OM) image of our JFET device fabricated on 285-nm-thick $\mathrm{SiO}_{2} / \mathrm{p}$-Si wafer. Red and blue dashed lines outline $\mathrm{MoS}_{2}$ and $\mathrm{MoTe}_{2}$ flake area, respectively. Scale bar $=5 \mu \mathrm{m}$. b Raman spectra obtained from red dot point of the overlapped region. c 3D schematic view of our $\mathrm{MoS}_{2}$ and $\mathrm{MoTe}_{2}$ junction device. Four heterojunction PN diode pairs can basically be formed (supporting information, Fig. S1) d 3D cross sectional views of p-MoTe2 channel (n-MoS2 gate) and n-MoS2 channel ( $p$ MoTe2 gate) JFET devices

supporting information in Figure S1). In addition, from the same structure, $\mathrm{p}$ - and n-type MISFET behavior was also confirmed along with large hysteresis in the transfer curve characteristics (Figure S2a).

Following the OM for the JFET structure (Figs. 1a and 2a inset), atomic force microscopy (AFM; Fig. 2a, b) and scanning kelvin probe microscopy (SKPM, Fig. 2c) were conducted probing a rectangular region of the same device, which contains four respective surfaces as shown in Fig. 2a: $\mathrm{SiO}_{2}$ substrate, $\mathrm{MoTe}_{2}$, $\mathrm{MoS}_{2}$, and overlay $\mathrm{MoS}_{2}$ on $\mathrm{MoTe}_{2}$. According to AFM results (image contrast), the thickness of $\mathrm{MoTe}_{2}$ and $\mathrm{MoS}_{2}$ channel appears to be $\sim 16$ and $6 \mathrm{~nm}$, respectively. According to SKPM results, the work functions of individual $\mathrm{MoTe}_{2}$ and $\mathrm{MoS}_{2}$ are quite the same, to be $4.54 \mathrm{eV}$, while that of $\mathrm{MoS}_{2}$ overlay on $\mathrm{MoTe}_{2}$ is slightly higher to be $4.56 \mathrm{eV}$. Slightly higher value is probably because $\mathrm{MoS}_{2}$ on $\mathrm{MoTe}_{2}$ is exempted from the effects of trap charges on $\mathrm{SiO}_{2}$ surface ${ }^{47,48}$ and also because of some electron charge transfer between the two TMDs. Based on SKPM data, we could expect and construct the band diagrams of $\mathrm{MoTe}_{2} / \mathrm{MoS}_{2} \mathrm{PN}$ junction, $\mathrm{MoS}_{2}$ n-channel, and $\mathrm{MoTe}_{2} \mathrm{p}$-channel as seen in Fig. $2 \mathrm{~d}-\mathrm{f}$, respectively. The $\mathrm{PN}$ junction should contain $\sim 0.3 \mathrm{~nm}$ vdW gap between $\mathrm{MoTe}_{2}$ and $\mathrm{MoS}_{2}$. Without gate bias, channel has almost no energy barrier but built-in potential energy $\left(q \Phi_{i}=\right.$ $0.02 \mathrm{eV}$ for $\mathrm{n}$-channel). When a reverse bias is applied to the $\mathrm{p}$-type gate of n-channel, the $\mathrm{MoS}_{2}$ channel should have energy barrier at the p-gated (overlapped) region (Fig. 2e), where the Fermi energy becomes located in the middle of the band gap indicating charge carrier depletion (for OFF state). Similarly, the $\mathrm{MoTe}_{2}$ channel has the energy barrier at the $\mathrm{n}$-gate region (Fig. 2f) under a reverse bias applied on p-channel. Without drain bias voltage, the band diagram with the barrier must be symmetric; however, it should become asymmetric under drain bias.

As expected from the in-plane direction band diagram (Fig. 2e, $f)$, n-channel JFET was experimentally demonstrated in Fig. 3a-f. Figure 3a shows an OM image of another JFET that is different from that of Fig. 1a but has a comparable channel thickness dimension: $\sim 12$ and $7 \mathrm{~nm}$, respectively, for $\mathrm{MoS}_{2}$ and $\mathrm{MoTe}_{2}$ channels. Output characteristics (drain current-drain voltage; $I_{D^{-}}$ $V_{\mathrm{DS}}$ ) of the device in Fig. 3d display three stages for typical transistors: linear (i), pinch-off (ii), and saturation-to-early effect (iii). Those three stages are well explained with schematic JFET cross-sections under, respectively, different $V_{D S}$ in Fig. $3 c$, while each material component of the device is identified by color in the schematic 3D view of Fig. 3b. Cross-section (i) in Fig. $3 \mathrm{c}$ shows a conducting channel under small $V_{D S}$ for linear regime $I_{D}$. As $V_{D S}$ increases toward more positive voltage, drain side experiences reverse bias with respect to the p-gate and asymmetric channel depletion (crosshatched area) takes place while source side maintains forward bias and channel opening. As $V_{D S}$ increases further, the $n$-channel reaches to pinch-off state (ii) and even channel length $(L)$ modulation (iii). Such channel length modulation causes shorter length $\left(L^{\prime}\right)$ and elevated current (deviated from saturation; early effect). This channel modulation was quite general in our JFET devices as observed from another JFET (supporting information Figure S3a). Figure $3 \mathrm{e}$ shows transfer characteristics (drain current-gate voltage; $I_{\mathrm{D}}-V_{\mathrm{GS}}$ ), where a good ON/OFF $I_{D}$ ratio of $5 \times 10^{4}$ and $S S$ of $\sim 100 \mathrm{mV} / \mathrm{dec}$ were observed. The gate leakage current $\left(I_{\mathrm{G}}\right)$ appears to increase with applied gate voltage $\left(V_{G S}\right)$ in Fig. 3e, and it is certainly understandable as 
(a)

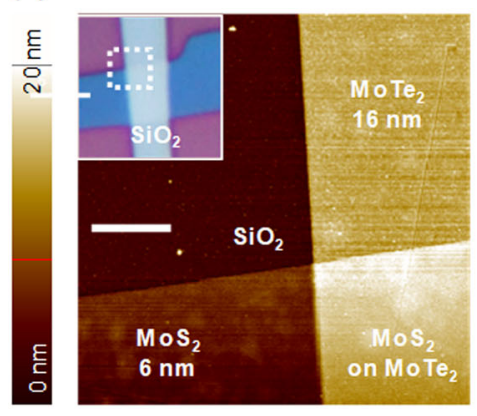

(b)

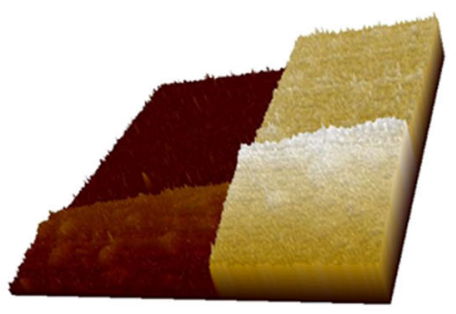

(c)

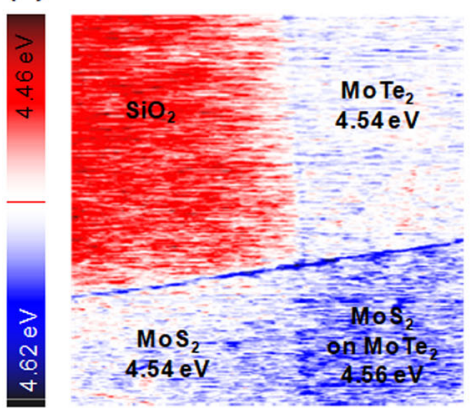

(d)

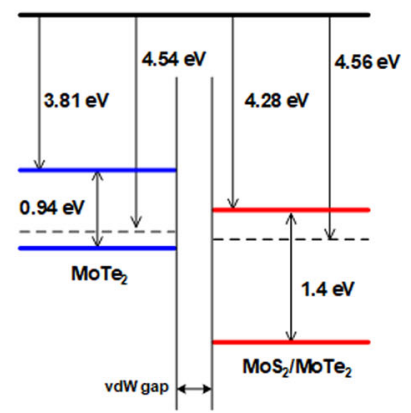

(e)

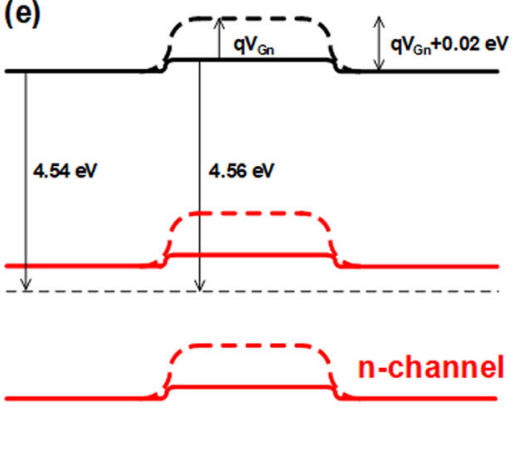

(f)

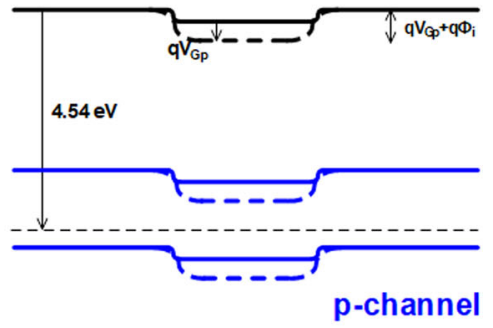

Fig. 2 Device band diagram by AFM and SKPM. a 2D and b 3D AFM images of the JFET structure. Scale bar $=1 \mu \mathrm{m}$. $\mathbf{c}$ SKPM image of the JFET. White dashed box in the inset of a indicates the scanning region for AFM and SKPM (a-c). Energy band diagrams of $\mathbf{d} \mathrm{MoTe}_{2} / \mathrm{MoS}_{2}$, e MoS $2 \mathrm{n}-$ channel, and $\mathbf{f} \mathrm{MoTe}_{2} \mathrm{p}$-channel, respectively. $V_{\mathrm{Gn}}$ and $V_{\mathrm{Gp}}$ means the gate biases, respectively, applied on $\mathrm{n}$ - and $\mathrm{p}$-channels

(a)

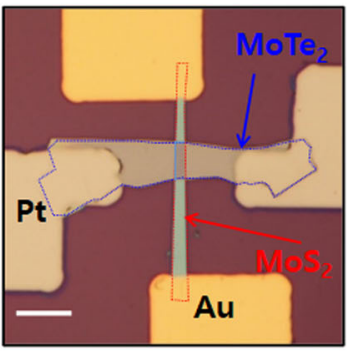

(d)

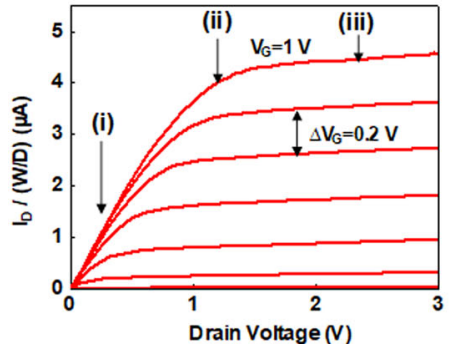

(b)

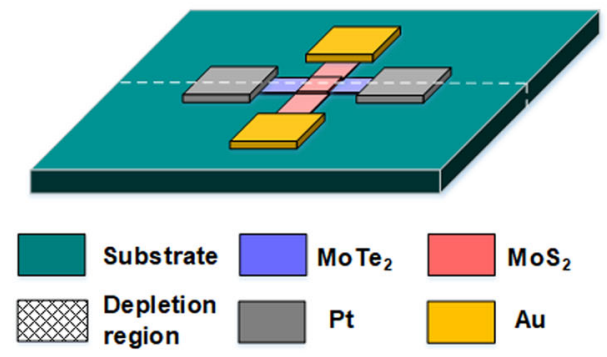

(e)

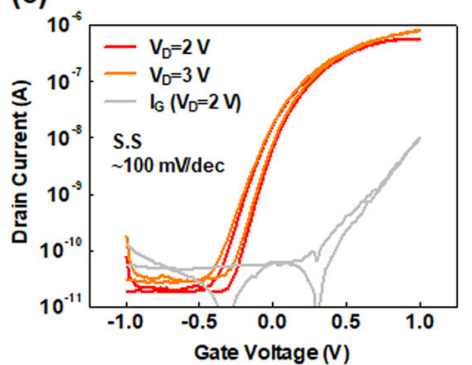

(c)

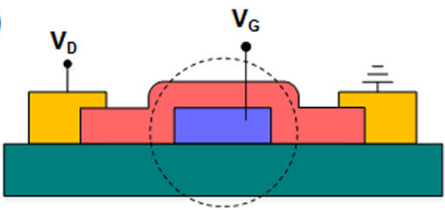

(i)
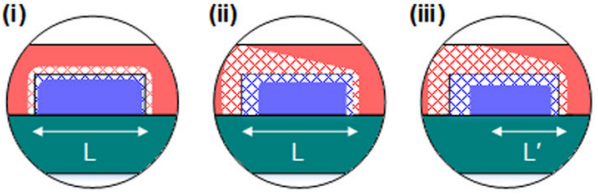

(f)

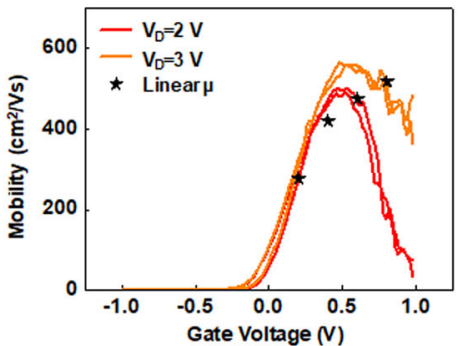

Fig. 3 n-channel of p-MoTe $2 / n-M_{2}$ JFET. a OM image of n-channel JFET. Scale bar $=10 \mu \mathrm{m}$. b Simple 3D schematic of our n-channel JFET. c $2 \mathrm{D}$ cross-section device views according to (i) small, (ii) pinch-off, and (iii) large $V_{\mathrm{DS}}$ as sectioned along with the white dashed line in $\mathbf{b}$ of our $\mathrm{n}$ channel device. d $I_{D}-V_{D S}$ output characteristics of $n$-channel JFET. e $I_{D}-V_{G S}$ transfer characteristics of $n$-channel JFET. f Mobility of our $n$ channel JFET. Red and orange lines indicate the saturation mobility and black stars indicate the linear mobility at different $V_{\mathrm{GS}}(0.2,0.4,0.6$, $0.8 \mathrm{~V}$, respectively)

forward bias-induced leakage that originates from the PN junction between $\mathrm{n}-\mathrm{MoS}_{2}$ and $\mathrm{p}-\mathrm{MoTe}_{2}$.

The saturation mobility of our JFET is also extracted from the same figure. Threshold voltage and peak saturation mobility appear to be $-0.2 \mathrm{~V}$ and $500-600 \mathrm{~cm}^{2} / \mathrm{V} \cdot \mathrm{s}$, respectively, according to Fig. $3 e, f$. The saturation mobility was driven from the following Eq. (1), which needs the information on carrier concentration, $N_{\mathrm{d}}$ and transconductance, $g_{\mathrm{m}}$, in $\mathrm{MoS}_{2}$ n-channel. We extract $g_{\mathrm{m}}$ plots 
(a)

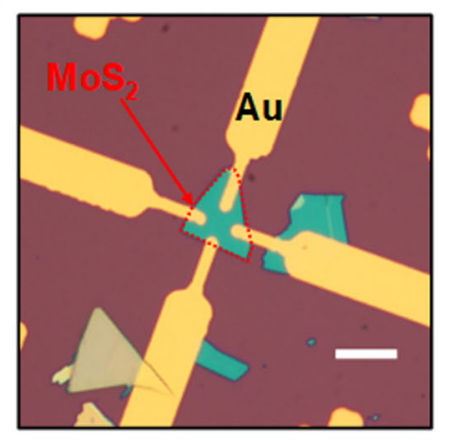

(c)
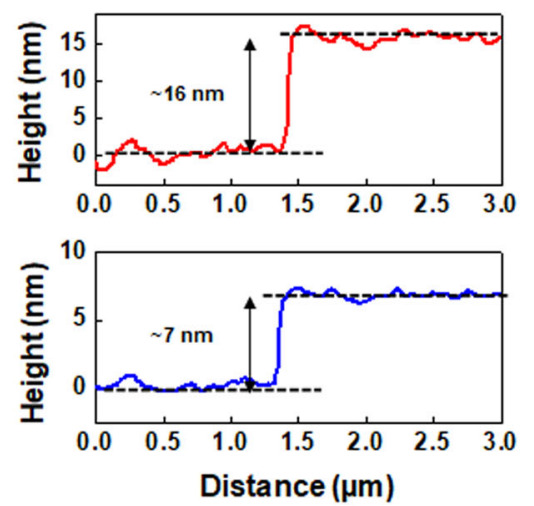

(b)

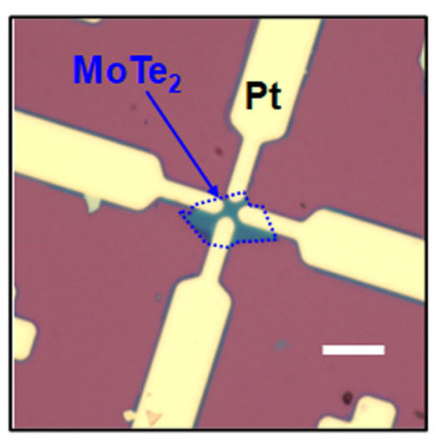

(d)
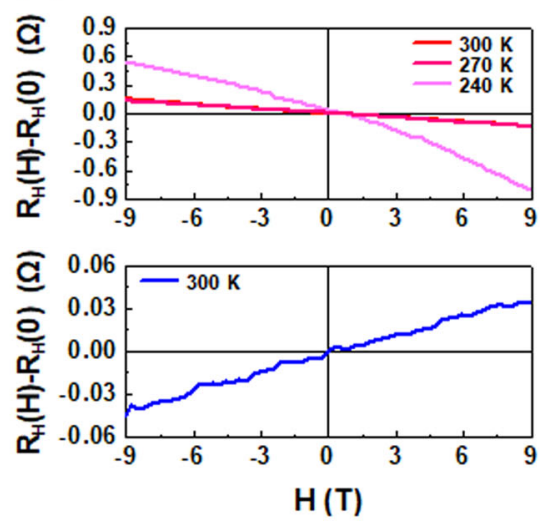

Fig. 4 Hall measurement of $\mathrm{MoS}_{2}$ and $\mathrm{MoTe}_{2}$. OM image of a $\mathrm{n}-\mathrm{MoS}_{2}$ and $\mathbf{b}$ p-MoTe $\mathrm{M}_{2}$ for four-probe Hall measurement. c Flake thickness profiles of $\mathrm{MoS}_{2}$ (red) and $\mathrm{MoTe}_{2}$ (blue) as obtained from AFM scanning, and $\mathbf{d} R_{H}(H)-R_{H}(0)$ data under the magnetic field $(H)$ for MoS 2 (top negative slopes) and $\mathrm{MoTe}_{2}$ (positive slope). Scale bar of $\mathbf{a}, \mathbf{b}=10 \mu \mathrm{m}$

from transfer curves as a function of $V_{\mathrm{GS}}$ in Fig. 3e.

$g_{\mathrm{m}}=\frac{d l_{\mathrm{D}}}{d V_{\mathrm{GS}}}=\frac{q N_{\mathrm{d}} \mu t W}{L}$

So the mobility can be calculated as follows,

$\mu=\frac{L g_{\mathrm{m}}}{q N_{\mathrm{d}} t W}$

where $N_{\mathrm{d}}$ is carrier density as number per $\mathrm{cm}^{3} ; q$ is an electronic charge; and $t, W$, and $L$ are the thickness, width, and length of the channel, respectively. Linear mobility can also be extracted out of the output characteristics at different $V_{\mathrm{GS}}$ in Fig. $3 d$, using the following simple Eq. (3) at small $V_{D S}$.

$\mu=\frac{L g_{\mathrm{d}}}{q N_{\mathrm{d}} t W}, \quad\left(g_{\mathrm{d}}=\frac{d l_{\mathrm{D}}}{d V_{\mathrm{DS}}}\right)$

The maximum linear mobility appears quite comparable to that of saturation regime.

For both estimations of saturation and linear mobilities, $N_{\mathrm{d}}$ value would be the most important information. In order to obtain $N_{\mathrm{d}}$ value at room temperature, we have actually attempted fourpoint van der Pauw Hall measurements with Au-contacting 16nm-thin $\mathrm{MoS}_{2}$ and Pt-contacting 7-nm-thin $\mathrm{MoTe}_{2}$. Figure $4 \mathrm{a}, \mathrm{b}$ show two OM images of our samples on $\mathrm{SiO}_{2} / \mathrm{p}$-Si substrate, while each thickness of the samples was measured by AFM scan as shown with the results of Fig. 4c. Although the sample shapes were not ideal symmetric in Fig. 4a, b, MoTe 2 and $\mathrm{MoS}_{2}$ samples displayed positive and negative slopes, respectively, under magnetic field $(H)$ sweep for relative magnetic resistance $\left[R_{H}(H)-\right.$ $\left.R_{H}(0)\right]$ vs. $H$ field plot in Fig. 4 d. Those slopes clearly identify or distinguish $\mathrm{p}$ - and $\mathrm{n}$-type conduction. According to the slope, hole and electron concentrations $\left(N_{\mathrm{a}}\right.$ and $\left.N_{\mathrm{d}}\right)$ were calculated to be $2.43 \times 10^{17}$ and $2.5 \times 10^{16} / \mathrm{cm}^{3}$ at $300 \mathrm{~K}$, respectively. Calculation details are in supporting information section.

All of our JFET devices displayed only a little hysteresis unlike MISFET (Figure S2a) because of small density charge traps at the vdW PN junction interface. Mobility plots in Fig. $3 f$ and transfer curves of Figure S2b exhibit a small hysteresis of $0.05-0.1 \mathrm{~V}$ whether the device is $\mathrm{n}$ - or $\mathrm{p}$-channel JFET (which is actually a single device working with both channels). Figure 5a shows our third JFET with p-channel, and in fact, this JFET has similar channel thickness of $\sim 10 \mathrm{~nm}$, which is comparable to that of n-channel JFET in Fig. 3a. Output curve characteristics in Fig. $5 \mathrm{~d}$ show the three $I_{D}$ regimes: linear (i), pinch-off (ii), and saturation (iii). At first glance, the p-channel $I_{D}$ output curves are comparable to those of $\mathrm{n}$-channel ones in Fig. 3d; however, it is recognized on detail observation that pinch-off stage appears slower in $\mathrm{MoTe}_{2} \mathrm{p}$ channel; saturation voltages $\left(V_{\mathrm{SAT}}=-1.5 \mathrm{~V}\right.$ for $\left.V_{\mathrm{GS}}=-1 \mathrm{~V}\right)$ of $\mathrm{p}$ channel are larger than those of $\mathrm{MoS}_{2} \mathrm{n}$-channel $\left(V_{\mathrm{SAT}}=\sim 0.8 \mathrm{~V}\right.$ for $V_{\mathrm{GS}}=1 \mathrm{~V}$ ). It is related to the hole carrier density of $\mathrm{p}$-channel, which is an order of magnitude larger than that of n-channel; the charge depletion of $\mathrm{p}$-channel is more difficult under the same $V_{\mathrm{GD}}\left(V_{\mathrm{GS}}-V_{\mathrm{DS}}\right)$ than that of $\mathrm{n}$-channel. Figure $5 \mathrm{~b}, \mathrm{c}$ present schematic $3 \mathrm{D}$ and cross-section views of the p-channel JFET. As shown in Fig. $5 c$, under a positive $V_{\mathrm{GD}} \mathrm{MoS}_{2}$ gate (reverse bias) is more readily depleted than $\mathrm{MoTe}_{2} \mathrm{p}$-channel, which needs further $V_{D S}$ for reaching to pinch-off. In Fig. 5e, our p-channel JFET shows an order of magnitude lower $I_{D}$ than that of $n$-channel one, along with inferior SS $(200 \mathrm{mV} / \mathrm{dec})$ and ON/OFF $I_{\mathrm{D}}$ ratio $\left(5 \times 10^{3}\right)$ to those of $n$-channel device. Difficult channel depletion or channel closing might be closely related to such inferiorities. According to Fig. $5 f$, the saturation $\left(13-14 \mathrm{~cm}^{2} / \mathrm{V} \cdot \mathrm{s}\right.$ as the peak mobility) and 
(a)
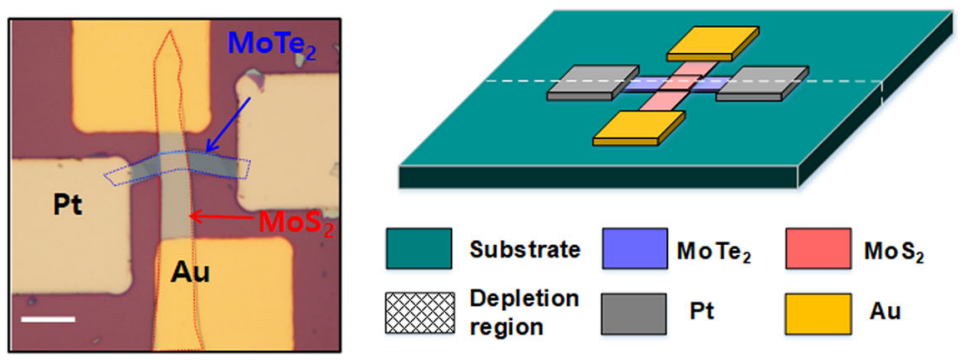

(d)

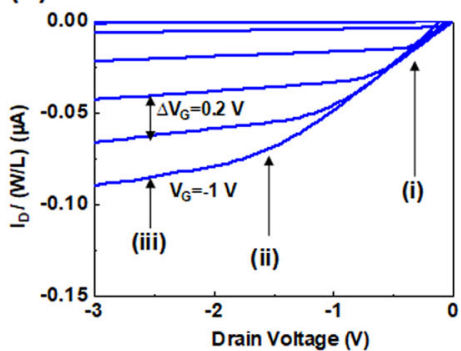

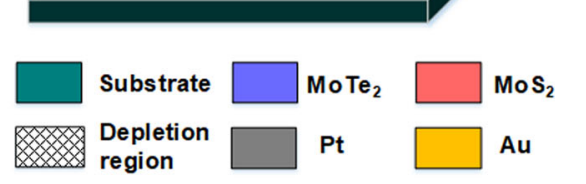

(b)

(e)

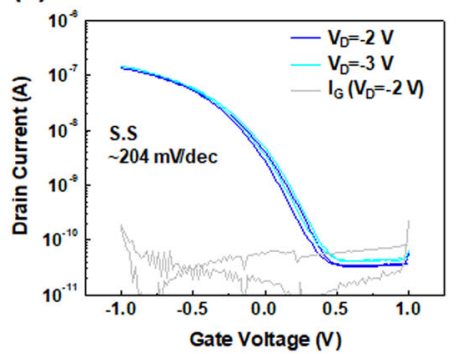

(c)

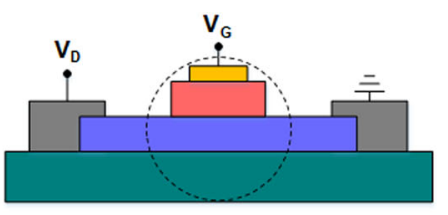

(i)

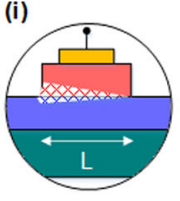

(ii)

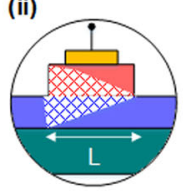

(iii)

(f)

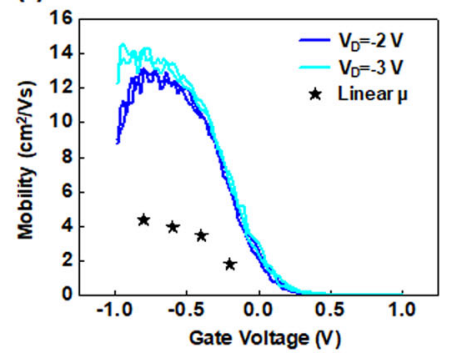

Fig. 5 p-channel of p-MoTe $2 / n-M_{2}$ JFET. a OM image of p-channel JFET. Scale bar $=10 \mu \mathrm{m}$. b Simple 3D schematic of our p-channel JFET. c $2 \mathrm{D}$ cross-section device views according to (i) small, (ii) pinch-off, and (iii) large $V_{D S}$ as sectioned along with the white dashed line in $\mathbf{b}$ of our $\mathrm{p}$ channel device. $\mathbf{d} I_{\mathrm{D}}-V_{\mathrm{DS}}$ output characteristics of $\mathrm{p}$-channel JFET. e $I_{\mathrm{D}}-V_{\mathrm{GS}}$ transfer characteristics of $\mathrm{p}$-channel JFET. f Mobility of our $\mathrm{p}$ channel JFET. Blue and skyblue lines indicate the saturation mobility and black stars indicate the linear mobility at different $V_{\mathrm{GS}}(-0.2,-0.4$, $-0.6,-0.8 \mathrm{~V}$, respectively)

linear mobilities $\left(4 \mathrm{~cm}^{2} / \mathrm{V} \cdot \mathrm{s}\right)$ of p-channel JFET appear comparable to the previous reports from $\mathrm{p}-\mathrm{MoTe}_{2}$ MISFETs $^{25}$ but much inferior to the values from $\mathrm{MoS}_{2}$ JFET. Impurity scattering due to an order of magnitude higher carrier concentration in the p-channel would be a main reason for the low mobility along with the intrinsic band structure of $\mathrm{MoTe}_{2}{ }^{49,50}$ Besides, we could also suspect the many traps at the $\mathrm{MoTe}_{2}$ channel/ $/ \mathrm{SiO}_{2}$ interface as another reason of such low mobility in respect of device geometry.

As our final device, $\mathrm{p}-\mathrm{WS} \mathrm{Se}_{2} / \mathrm{n}-\mathrm{MoS}_{2}$ JFET was fabricated on purpose to confirm that any $\mathrm{p}$-TMD/n-TMD JFET generally works in principle; $\mathrm{p}$-TMD works as gate for $\mathrm{n}-\mathrm{TMD}$ channel while $\mathrm{n}$-TMD does as gate for p-TMD channel. Figure $6 a, b$, respectively, show the $\mathrm{OM}$ and schematic 3D view of the JFET, where Au contact was used in common for both $\mathrm{p}-\mathrm{WSe}_{2}$ and $\mathrm{n}-\mathrm{MoS}_{2}$ channel FETs. Figure $6 c$ displays Raman spectra from both flakes as obtained at once by probing the overlaid position (red dot in Fig. 6a). According to output curve characteristics of Fig. $6 \mathrm{~d}, \mathrm{p}$ - and nchannel JFETs operate well again, although the contact resistance between $\mathrm{Au}$ and $\mathrm{p}-\mathrm{WSe}_{2}$ appears serious. Owing to such shortcoming in contact resistance, $\mathrm{p}-\mathrm{WSe}_{2}$ JFET shows its inferior $I-V$ characteristics with a few $\mathrm{nA}$ of $\mathrm{ON}$ state, and the inferior conductance of $\mathrm{p}$-channel $\mathrm{WSe}_{2}$ results in its insufficient gating for $n-M_{2}$ JFET. Hence, n-MoS 2 JFET in $\mathrm{p}-\mathrm{WSe}_{2} / \mathrm{n}-\mathrm{MoS}_{2}$ system displays an order of magnitude lower ON state $I_{D}$ than that of $p$ $\mathrm{MoTe}_{2} / \mathrm{n}-\mathrm{MoS}_{2}$ JFET case, as seen in the output and transfer characteristics of Fig. $6 \mathrm{~d}$, e. However, this demonstration of $\mathrm{p}$ WSe $2 / n-M_{2}$ JFET device still supports that any $\mathrm{p}-\mathrm{TMD} / \mathrm{n}-\mathrm{TMD}$ JFET generally works in principle using both channels. (Figure S4 shows AFM thickness profile of $\mathrm{p}-\mathrm{WSe}_{2}$ and $\mathrm{n}-\mathrm{MoS}_{2}$ flakes in JFET.)

In summary, we have fabricated vdW JFETs as an in-plane current device with heterojunction between semiconducting $p$ $\mathrm{MoTe}_{2}$ and $\mathrm{n}-\mathrm{MoS}_{2}$ TMDs. Since this vdW JFET would have lowdensity traps at the vdW interface when p-type material plays as a gate for $\mathrm{n}$-channel and vice versa, little hysteresis of 0.05-0.1 V and good SS of $\sim 100 \mathrm{mV} / \mathrm{dec}$ were achieved. Easy saturation was observed as another advantage over 2D MISFETs, exhibiting early pinch-off at $\sim 1 \mathrm{~V}$. Operational gate voltage for threshold was near $0 \mathrm{~V}$ and the highest mobility reaches to $\sim 500 \mathrm{~cm}^{2} / \mathrm{V} \cdot \mathrm{s}$ for $\mathrm{n}$ channel JFET with $\mathrm{MoS}_{2}$ channel while p-channel JFET with $\mathrm{MoTe}_{2}$ appears much lower by more than an order of magnitude $\left(\sim 13 \mathrm{~cm}^{2} / \mathrm{V} \cdot \mathrm{s}\right)$. For $1 \mathrm{~V}$ JFET operation, our highest ON/OFF current ratio was observed to be $\sim 10^{4}$. The operation of both channel JFETs with ultrathin vdW 2D TMDs is regarded unique and different from that of general 3D JFETs and MISFETs in the principle that two opposite ( $p$ - and $n-$ ) channels can be used as gate for each other. We again confirmed the principle through another $\mathrm{p}-\mathrm{TMD} / \mathrm{n}-\mathrm{TMD}$ JFET ( $\mathrm{p}-\mathrm{WSe}_{2} / \mathrm{n}-\mathrm{MoS}_{2}$ junction). We thus conclude that our 2D-like ultrathin channel JFET is fresh enough in its operating principle, structure, and fabrication simplicity to influence the future of $2 \mathrm{D}$ semiconductor-based nanoelectronics.

\section{METHODS}

\section{Device fabrication}

$\mathrm{SiO}_{2} / \mathrm{p}$-Si wafer (285-nm thick) was chosen as JFET device substrate. The substrates were cleaned in acetone and methyl alcohol by ultrasonicator. For $\mathrm{p}-\mathrm{MoTe}_{2} / \mathrm{n}-\mathrm{MoS}_{2}$ JFETs, a-MoTe $e_{2}$ nanosheet (mechanically exfoliated from bulk crystals using polydimethylsiloxane) was transferred onto the substrate. Fifty-nm (Pt) ohmic electrodes for $\mathrm{MoTe}_{2}$ were patterned by photolithography process and deposited by direct current (DC) magnetron sputtering system. In the next step, $\mathrm{MoS}_{2}$ nanoflake was transferred onto the $\mathrm{MoTe}_{2}$ flake forming vdW junction. Fifty-nm (Au) ohmic electrodes for $\mathrm{MoS}_{2}$ were also patterned in the same way. For $\mathrm{p}-\mathrm{WSe} \mathrm{e}_{2} / \mathrm{n}-\mathrm{MoS}_{2}$ JFETs, $\mathrm{WSe}_{2}$ nanosheet was transferred onto the substrate and then $\mathrm{MoS}_{2}$ nanoflake was crossed over contacting $\mathrm{WSe}_{2}$ flake. Au ohmic electrodes for both $\mathrm{WSe}_{2}$ and $\mathrm{MoS}_{2}$ were patterned by photolithography process and DC magnetron sputter deposition.

\section{Characterization}

All nanoflakes were measured for thickness information with AFM (Nanowizard I, JPK Instrument). SKPM measurements were performed 
(a)

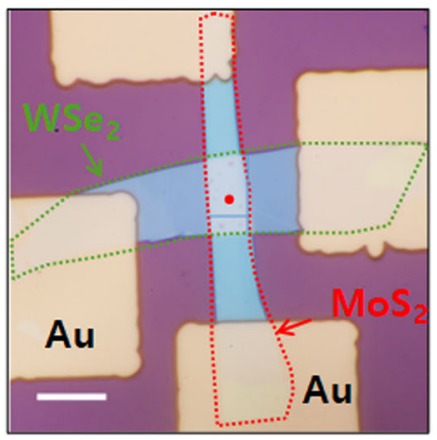

(b)

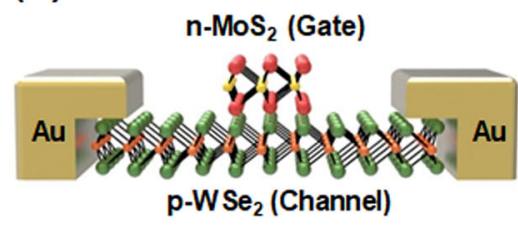

$\mathrm{n}-\mathrm{MoS}_{2}$ (Channel)

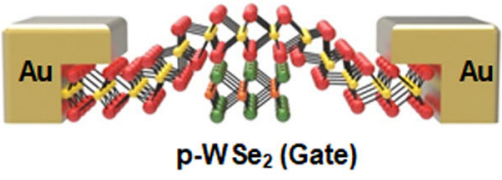

(c)

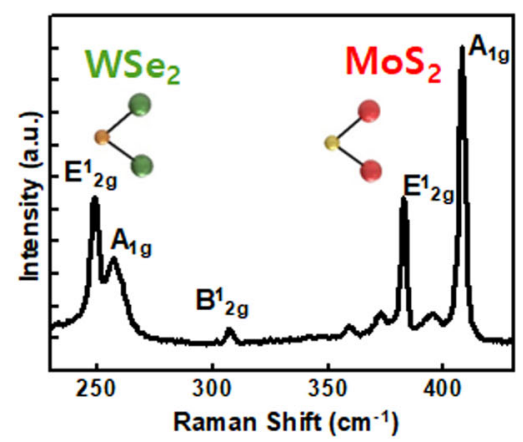

(d)

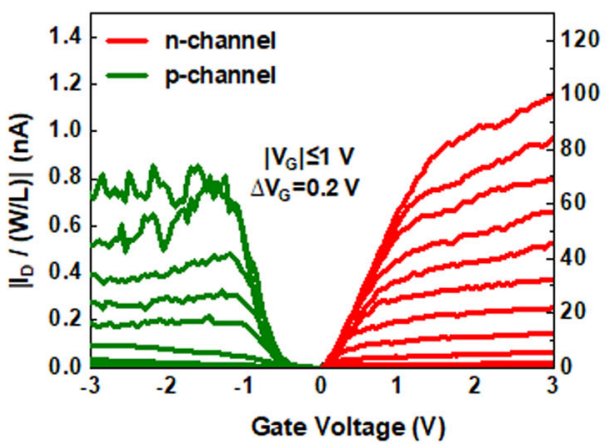

(e)

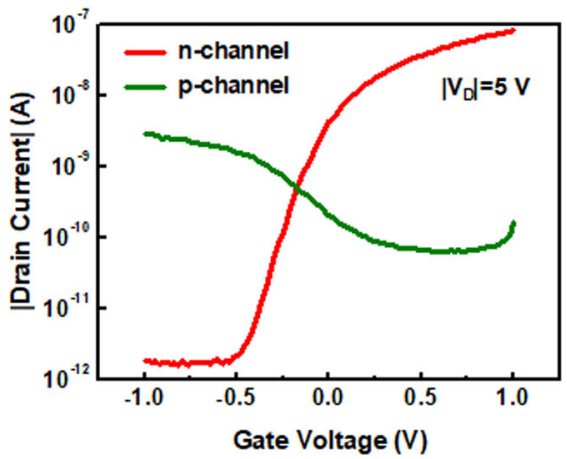

Fig. 6 p-WSe $2 / n-M_{2} S_{2}$ JFET. a OM image of p-WSe $/ n-M_{2} S_{2}$ JFET on $\mathrm{SiO}_{2} / \mathrm{p}-\mathrm{Si}$. Scale bar $=10 \mu \mathrm{m}$. $\mathbf{b}$ 3D schematic cross-sections of $\mathrm{p}-\mathrm{WS} \mathrm{e}_{2}$ and $\mathrm{n}-\mathrm{MoS}_{2}$ channel JFET. Au was used as contact metal for both channels. c Raman spectra of WSe $\mathrm{Wnd} \mathrm{MoS}_{2}$ as obtained by probing the red spot in a. d $I_{D}-V_{D S}$ output characteristics of $\mathrm{p}-\mathrm{WSe}_{2}$ and $n-\mathrm{MoS}_{2}$ channel JFET. e $I_{\mathrm{D}}-V_{\mathrm{GS}}$ transfer characteristics of $\mathrm{p}-\mathrm{WS} \mathrm{e}_{2}$ and $\mathrm{n}-\mathrm{MoS}_{2}$ channel JFET

using Park Systems XE7 with non-contact mode. In SKPM imaging, we applied an alternating current (AC) bias voltage of $1.5 \mathrm{~V}$ with a frequency of $17 \mathrm{kHz}$ to Au-coated tip (PPP-NCSTAu, nanosensors). Device electrical characteristics (I- $V$ curve of diodes and transfer and output characteristics of transistors) were performed in the dark at room temperature using a semiconductor parameter analyzer (HP4155C, Agilent Technologies). The Hall measurement for carrier density estimation of $\mathrm{MoS}_{2}$ and $\mathrm{MoTe}_{2}$ nanoflakes on $\mathrm{SiO}_{2} / \mathrm{p}^{+}$-Si substrate were conducted by applying an $\mathrm{AC}$ current of $0.4 \mathrm{~Hz}$ and $0.1 \mathrm{~mA}$ as input signal frequency and amplitude, respectively, under the magnetic field $(H)$ sweep from -9 to $9 \mathrm{~T}$ using physical property measurement system.

\section{DATA AVAILABILITY}

The authors confirm that the data supporting the findings of this study are available within the article and its supplementary materials.

\section{ACKNOWLEDGEMENTS}

The authors acknowledge the financial support from NRF (NRL program: Grant No. 2017R1A2A1A05001278, SRC program: Grant No.2017R1A5A1014862, vdWMRC center). J.Y.L. acknowledges the tuition support from the Hyundai Motor Chung Mong-Koo Foundation.

\section{AUTHOR CONTRIBUTIONS}

J.Y.L. conducted all the experiments and analysis; M.K., Y.Y., and T.K. supported the SKPM measurement; Y.J. and H.G.S. helped device fabrications; K.R.K., J.Y.M., and Y.J.C. helped Hall measurement; S.Y. helped with 3D figure scheme techniques; S.I. designed whole-device experiments.

\section{ADDITIONAL INFORMATION}

Supplementary information accompanies the paper on the npj 2D Materials and Applications website (https://doi.org/10.1038/s41699-018-0082-2).

Competing interests: The authors declare no competing interests.

Publisher's note: Springer Nature remains neutral with regard to jurisdictional claims in published maps and institutional affiliations.

\section{REFERENCES}

1. Zhou, L. et al. Large-area synthesis of high-quality uniform few-layer MoTe $\mathrm{M}_{2} . \mathrm{J}$. Am. Chem. Soc. 137, 11892-11895 (2015).

2. Keum, D. H. et al. Bandgap opening in few-layered monoclinic MoTe 2 . Nat. Phys. 11, 482-486 (2015).

3. Fathipour, S. et al. Exfoliated multilayer $\mathrm{MoTe}_{2}$ field-effect transistors. Appl. Phys. Lett. 105, 2014-2017 (2014).

4. Chhowalla, M. et al. The chemistry of two-dimensional layered transition metal dichalcogenide nanosheets. Nat. Chem. 5, 263-275 (2013).

5. Qi, Y. et al. Superconductivity in Weyl semimetal candidate MoTe 2 . Nat. Commun. 7, 11038 (2016)

6. Ruppert, C., Aslan, O. B. \& Heinz, T. F. Optical properties and band gap of singleand few-layer MoTe $\mathrm{M}_{2}$ crystals. Nano Lett. 14, 6231-6236 (2014).

7. Radisavljevic, B., Radenovic, A., Brivio, J., Giacometti, V. \& Kis, A. Single-layer $\mathrm{MoS}_{2}$ transistors. Nat. Nanotechnol. 6, 147-150 (2011).

8. Das, S., Chen, H.-Y., Penumatcha, A. V. \& Appenzeller, J. High performance multilayer $\mathrm{MoS}_{2}$ transistors with scandium contacts. Nano Lett. 13, 100-105 (2013).

9. Lee, H. S. et al. $\mathrm{MoS}_{2}$ nanosheet phototransistors with thickness-modulated optical energy gap. Nano Lett. 12, 3695-3700 (2012).

10. Choi, K. et al. Direct imprinting of $\mathrm{MoS}_{2}$ flakes on a patterned gate for nanosheet transistors. J. Mater. Chem. C 1, 7803 (2013).

11. Lezama, I. G. et al. Indirect-to-direct band gap crossover in few-layer MoTe $\mathrm{Mano}_{2}$ Lett. 15, 2336-2342 (2015). 
12. Lee, H. S. et al. Metal semiconductor field-effect transistor with $\operatorname{MoS}_{2} /$ conducting $\mathrm{NiO}_{\mathrm{x}}$ van der Waals Schottky interface for intrinsic high mobility and photoswitching speed. ACS Nano 9, 8312-8320 (2015).

13. Wang, Q. H., Kalantar-Zadeh, K., Kis, A., Coleman, J. N. \& Strano, M. S. Electronics and optoelectronics of two-dimensional transition metal dichalcogenides. Nat. Nanotechnol. 7, 699-712 (2012).

14. Yin, Z. et al. Single-layer $\mathrm{MoS}_{2}$ phototransistors. ACS Nano 6, 74-80 (2012).

15. Fang, $H$. et al. High-performance single layered $W \mathrm{Se}_{2}$ P-FETs with chemically doped contacts. Nano Lett. 12, 3788-3792 (2012).

16. Lopez-Sanchez, O., Lembke, D., Kayci, M., Radenovic, A. \& Kis, A. Ultrasensitive photodetectors based on monolayer MoS . Nat. Nanotechnol. 8, 497-501 (2013).

17. Liu, W. et al. Role of metal contacts in designing high-performance monolayer $n$ type WSe $\mathrm{S}_{2}$ field effect transistors. Nano Lett. 13, 1983-1990 (2013).

18. Wang, $\mathrm{H}$. et al. Integrated circuits based on bilayer $\mathrm{MoS}_{2}$ transistors. Nano Lett 12, 4674-4680 (2012)

19. Radisavljevic, B., Whitwick, M. B. \& Kis, A. Integrated circuits and logic operations based on single-layer MoS $_{2}$. ACS Nano 5, 9934-9938 (2011).

20. Di Bartolomeo, A. et al. Hysteresis in the transfer characteristics of $\mathrm{MoS}_{2}$ transistors. 2D Mater. 5, 015014 (2017).

21. Li, C. et al. Low sub-threshold swing realization with contacts of graphene/h-BN/ $\mathrm{MoS}_{2}$ heterostructures in $\mathrm{MoS}_{2}$ transistors. Appl. Phys. Lett. 111, 193502 (2017).

22. Liu, N. et al. Improving the stability of high-performance multilayer $\mathrm{MoS}_{2}$ fieldeffect transistors. ACS Appl. Mater. Interfaces 9, 42943-42950 (2017)

23. Lim, J. Y. et al. Homogeneous 2D MoTe, P-N junctions and CMOS inverters formed by atomic-layer-deposition-induced doping. Adv. Mater. 29, 1701798 (2017).

24. Jin, Y. et al. Van der Waals homojunction: ideal p-n diode behavior in $\mathrm{MoSe}_{2}$. Adv Mater. 27, 5534-5540 (2015).

25. Pezeshki, A. et al. Static and dynamic performance of complementary inverters based on nanosheet a-MoTe 2 p-channel and $\mathrm{MoS}_{2} \mathrm{n}$-channel transistors. ACS Nano 10, 1118-1125 (2016)

26. Tosun, $\mathrm{M}$. et al. High-gain inverters based on $\mathrm{WSe}_{2}$ complementary field-effect transistors. ACS Nano 8, 4948-4953 (2014).

27. $\mathrm{Yu}$, L. et al. High-performance WSe $\mathrm{H}_{2}$ complementary metal oxide semiconductor technology and integrated circuits. Nano Lett. 15, 4928-4934 (2015).

28. Das, S., Dubey, M. \& Roelofs, A. High gain, low noise, fully complementary logic inverter based on bi-layer WSe ${ }_{2}$ field effect transistors. Appl. Phys. Lett. 105, 1-6 (2014).

29. Lee, C.-H. et al. Atomically thin $\mathrm{p}-\mathrm{n}$ junctions with van der Waals heterointerfaces. Nat. Nanotechnol. 9, 676-681 (2014).

30. Zhang, K. et al. Interlayer transition and infrared photodetection in atomically thin type-II $\mathrm{MoTe}_{2} / \mathrm{MoS}_{2}$ van der Waals heterostructures. ACS Nano 10, 3852-3858 (2016).

31. Wang, B. et al. Enhanced current rectification and self-powered photoresponse in multilayer p-MoTe $/ \mathrm{n}-\mathrm{MoS}_{2}$ van der Waals Heterojunctions. Nanoscale 9, 10733-10740 (2017).

32. Wang, F. et al. Tunable GaTe-MoS 2 van der Waals $\mathrm{p}-\mathrm{n}$ junctions with novel optoelectronic performance. Nano Lett. 15, 7558-7566 (2015).

33. Doan, M.-H. et al. Charge transport in $\mathrm{MoS}_{2} / \mathrm{WSe}_{2}$ van der Waals heterostructure with tunable inversion layer. ACS Nano 11, 3832-3840 (2017).

34. Cho, A. J. et al. Electric and photovoltaic characteristics of a multi-layer $\operatorname{ReS}_{2} /$ $\mathrm{ReSe}_{2}$ heterostructure. Nano Lett. 14, 5590-5597 (2014).
35. Cheng, R. et al. Electroluminescence and photocurrent generation from atomically sharp WSe $\mathrm{WoS}_{2}$ heterojunction p-n diodes. Nano Lett. 14, 5590 (2014).

36. Ahn, J. et al. Transition metal dichalcogenide heterojunction PN diode toward ultimate photovoltaic benefits. 2D Mater. 3, 045011 (2016)

37. Ross, J. S. et al. Interlayer exciton optoelectronics in a $2 \mathrm{D}$ heterostructure $\mathrm{p}-\mathrm{n}$ junction. Nano Lett. 17, 638-643 (2017).

38. Jariwala, D. et al. Large-area, low-voltage, antiambipolar heterojunctions from solution-processed semiconductors. Nano Lett. 15, 416-421 (2015).

39. Jariwala, D. et al. Gate-tunable carbon nanotube- $\mathrm{MoS}_{2}$ heterojunction p-n diode. Proc. Natl. Acad. Sci. 110, 18076-18080 (2013).

40. Jariwala, D. et al. Hybrid, gate-tunable, van der Waals $p-n$ heterojunctions from pentacene and $\mathrm{MoS}_{2}$. Nano Lett. 16, 497-503 (2016).

41. Furchi, M. M., Pospischil, A., Libisch, F., Burgdörfer, J. \& Mueller, T. Photovoltaic effect in an electrically tunable van der Waals heterojunction. Nano Lett. 14 4785-4791 (2014).

42. Roy, T. et al. Dual-gated $\mathrm{MoS}_{2} / \mathrm{WSe}_{2}$ van der Waals tunnel diodes and transistors. ACS Nano 9, 2071-2079 (2015).

43. Kim, J., Mastro, M. A., Tadjer, M. J. \& Kim, J. Heterostructure WSe $\mathrm{S}_{2}-\mathrm{Ga}_{2} \mathrm{O}_{3}$ junction field-effect transistor for low-dimensional high-power electronics. ACS Appl. Mater. Interfaces 10, 29724-29729 (2018).

44. Cheng, R. et al. High-performance, multifunctional devices based on asymmetric van der Waals heterostructure. Nat. Electo. 1, 356-361 (2018).

45. Jeon, P. J. et al. Black phosphorus-zinc oxide nanomaterial heterojunction for $p-n$ diode and junction field-effect transistor. Nano Lett. 16, 1293-1298 (2016).

46. Shin, H. G. et al. Vertical and in-plane current devices using $\mathrm{NbS}_{2} / \mathrm{n}-\mathrm{MoS}_{2}$ van der Waals Schottky junction and graphene contact. Nano Lett. 18, 1937-1945 (2018)

47. Li, Y., Xu, C. Y. \& Zhen, L. Surface potential and interlayer screening effects of fewlayer $\mathrm{MoS}_{2}$ nanoflakes. Appl. Phys. Lett. 102, 3-7 (2013).

48. Castellanos-Gomez, A. et al. Electric-field screening in atomically thin layers of $\mathrm{MoS}_{2}$ : the role of interlayer coupling. Adv. Mater. 25, 899-903 (2013).

49. Lezama, I. G. et al. Surface transport and band gap structure of exfoliated $2 \mathrm{H}$ $\mathrm{MoTe}_{2}$ crystals. 2D Mater. 1, 21002 (2014).

50. Muller, R. S., Kamins, T. I. \& Chan, M. Device Electronics for Integrated Circuits (John Wiley \& Sons, Inc., New York, NY, 2003).

Open Access This article is licensed under a Creative Commons Attribution 4.0 International License, which permits use, sharing, adaptation, distribution and reproduction in any medium or format, as long as you give appropriate credit to the original author(s) and the source, provide a link to the Creative Commons license, and indicate if changes were made. The images or other third party material in this article are included in the article's Creative Commons license, unless indicated otherwise in a credit line to the material. If material is not included in the article's Creative Commons license and your intended use is not permitted by statutory regulation or exceeds the permitted use, you will need to obtain permission directly from the copyright holder. To view a copy of this license, visit http://creativecommons. org/licenses/by/4.0/.

(c) The Author(s) 2018 